NASA/TM-1998-207423

NASA

\title{
Monte Carlo Computational Modeling of the Energy Dependence of Atomic Oxygen Undercutting of Protected Polymers
}

Bruce A. Banks

Lewis Research Center, Cleveland, Ohio

Thomas J. Stueber

NYMA, Inc., Brook Park, Ohio

Mary Jo Norris

Cleveland State University, Cleveland, Ohio

Prepared for the

Fourth International Space Conference on Protection of Materials and Structures from the LEO Space Environment sponsored by the University of Toronto Institute for Aerospace Studies Toronto, Canada, April 23-24, 1998

National Aeronautics and

Space Administration

Lewis Research Center 
Available from

NASA Center for Aerospace Information

7121 Standard Drive

Hanover, MD 21076

Price Code: $\mathrm{A} 03$

National Technical Information Service 5287 Port Royal Road Springfield, VA 22100 Price Code: A03 


\title{
MONTE CARLO COMPUTATIONAL MODELING OF THE ENERGY DEPENDENCE OF ATOMIC OXYGEN UNDERCUTTING OF PROTECTED POLYMERS
}

\author{
Bruce A. Banks \\ National Aeronautics and Space Administration \\ Lewis Research Center \\ Cleveland, Ohio 44135 \\ Thomas J. Stueber \\ NYMA, Inc. \\ Brook Park, Ohio 44142 \\ and \\ Mary Jo Norris \\ Cleveland State University \\ Cleveland, Ohio 44115
}

\begin{abstract}
SUMMARY
A Monte Carlo computational model has been developed which simulates atomic oxygen attack of protected polymers at defect sites in the protective coatings. The parameters defining how atomic oxygen interacts with polymers and protective coatings as well as the scattering processes which occur have been optimized to replicate experimental results observed from protected polyimide Kapton on the Long Duration Exposure Facility (LDEF) mission. Computational prediction of atomic oxygen undercutting at defect sites in protective coatings for various arrival energies was investigated. The atomic oxygen undercutting energy dependence predictions enable one to predict mass loss that would occur in low Earth orbit, based on lower energy ground laboratory atomic oxygen beam systems. Results of computational model prediction of undercut cavity size as a function of energy and defect size will be presented to provide insight into expected in-space mass loss of protected polymers with protective coating defects based on lower energy ground laboratory testing.
\end{abstract}

\section{INTRODUCTION}

Atomic oxygen in low Earth orbit (LEO) has been found to readily react with most hydrocarbon and fluoropolymer materials. ${ }^{1-5}$ The rates of oxidation of most polymers have been sufficiently high that concerns about long term durability have prompted efforts to develop polymers which are resistant to atomic oxygen attack, ${ }^{6}$ or atomic oxygen durable protective coatings which would prevent the underlying material from being attacked. ${ }^{2.7-10}$ Efforts to evaluate the effectiveness of atomic oxygen protective coatings on polymers have indicated in both ground laboratory and in-space testing that atomic oxygen is capable of entering defect sites (pin windows and cracks) in protective coatings to produce under-cutting through oxidation much greater in extent than the initial size of the defect. ${ }^{11-14}$ Even though pin window and scratch defects in protective coatings may represent a small fractional area of a polymer surface in LEO, undercutting oxidation may result in structural failure of the polymer. The extent of oxidation can ultimately be sufficiently large to allow interconnection of the undercut oxidation sites. Although ground laboratory facilities can be used to simulate the oxidizing effects of LEO atomic oxygen exposure, differences in the arriving atomic oxygen energies between ground facilities and LEO cause some concern about applying the durability information derived from the ground laboratory to the space environment. Because atomic oxygen reaction probability is strongly dependent upon its impact energy, differences between the ground laboratory impact energies and in-space energies must be taken into account to reliably predict in-space durability based on ground laboratory testing. ${ }^{15}$ To a great extent, the energy differences have been compensated for by using effective fluence when measuring effects of atomic oxygen erosion. The effective fluence of atomic oxygen is the number of 
impinging atoms per area that would be required in LEO to produce the amount of erosion observed in an arbitrary energy ground laboratory system. Thus, for ground laboratory systems which produce atomic oxygen energies below those observed in LEO, many more actual atoms are required to produce the same amount of erosion that would occur in LEO.

Confidence in prediction of in-space durability based on ground laboratory testing can be improved by quantifying the dependence of undercutting oxidation on arriving oxygen atom energy. This investigation uses a Monte Carlo computational modeling technique to compare the differences in undercutting oxidation resulting from atomic oxygen arrival at various energies. By comparison of the volume loss or mass loss (by multiplying by the density) as a function of atomic oxygen arrival energy for the same size defect in the protective coating, mass loss in space can be projected at whatever appropriate LEO energy exists, based on known energy ground laboratory data. Because a realistic protective coating has a distribution of defect sizes, undercutting dependence upon defect size must be taken into account.

This paper addresses the prediction of in-space mass loss under the conditions of fixed direction atomic oxygen attack compared to ground laboratory mass loss at different energies for thick (polymer thickness exceeds maximum erosion depth) polymers protected by thin film coatings which contain defects, that allow atomic oxygen exposure of the underlying polymer.

\section{PROCEDURE}

A Monte Carlo computational model was used to simulate the erosion effects of atomic oxygen interacting with protected polymers at various energies for this investigation. ${ }^{16-18}$ The computational model simulates a cross-section of a portion of polymer being exposed to simulated atomic oxygen impact at the site of a defect in the protective coating. Because the model is a two-dimensional model, each defect is considered a crack or scratch type defect as opposed to a pin-window. The polymer is simulated by an orthogonal array of up to 500 $\times 500$ cells. The cells represent groups of polymer atoms which can potentially be oxidized. The model is capable of simulating monoenergetic or distributed energy atomic oxygen arrival as well as angularly distributed atomic oxygen arrival. Such angularly distributed arrival occurs as a result of the combined effects of Maxwellian distribution of thermal search atoms and velocity components associated with orbital inclination. ${ }^{18}$ The computational model simulates the effect of atomic oxygen attack by allowing model atomic oxygen atoms to enter the defect site and impinge upon the underlying polymer. Three possible events can occur as a result of the atomic oxygen impact on a representative polymer model cell. An atomic oxygen reaction can occur which would result in the removal of the cell, the oxygen can scatter from the cell without reaction, or the oxygen can recombine to form nonreactive $\mathrm{O}_{2}$. The probability of atomic oxygen reacting with the polymer cell is dependent upon the impact energy of the atom as well as local angle of attack. Atomic oxygen which does not react and then scatters from the polymer surface can do so in a manner which ranges from specular to diffuse. The atomic oxygen which leaves the surface can also partially thermally accommodate to the polymer surface such that it leaves with a lower energy than the initial impact energy. Scattered unreacted atomic oxygen atoms are free to potentially react at other locations within the undercut cavities or be ejected from the initial defect entrance. The interaction parameters used for this investigation are listed in table I. Quantification of the parameters is based on the results of an effort to optimize the Monte Carlo modeling interaction parameters in order to produce computational results in agreement with experimental observations of atomic oxygen undercutting observed on protected polyimide Kapton samples returned from the Long Duration Exposure Facility.

The actual parameters used for the simulation of ground laboratory atomic oxygen exposure would differ from table I in that the initial impact reaction probability $(0.11)$ may be different if the initial energy of the atomic oxygen was not $4.5 \mathrm{eV}$. The initial reaction probability is based on the actual atom energy and the activation energy. For ground laboratory atomic oxygen exposures, the initial impact energy assumed for the model is whatever energy is being considered as representative of the ground laboratory facility.

Because each Monte Carlo atom represents many actual atoms and each Monte Carlo cell represents a measurable cross-sectional square of polymer, one must calibrate the Monte Carlo simulated exposure to draw meaningful conclusions from the predicted undercutting erosion. 
The computation of how many Monte Carlo atoms should enter through the defect to represent an actual in-space fluence was accomplished using equation (1):

$$
\mathbf{N}=\frac{M}{H} \frac{E F}{D L} \mathbf{W}
$$

where

$\mathrm{N}$ number of Monte Carlo atoms needed to simulate an effective fluence of $\mathrm{F}$ (atoms)

$M$ number of atoms for wide defect (atoms) where width of defect $\gg$ thickness of polyimide

E space erosion yield $\left(\mathrm{cm}^{3} / \mathrm{atom}\right)$

F fluence in space $\left(\right.$ atoms $\left./ \mathrm{cm}^{2}\right)$

W narrow crack width (cell)

$\mathrm{H}$ wide crack width (cell)

D Monte Carlo thickness lost (cell)

$\mathrm{L}$ length of the edge of a cell $(\mathrm{cm} / \mathrm{cell})$

This calibration technique is shown schematically in figure 1 . Because the Monte Carlo model requires the presence of a protective coating with a crack defect, the computational calibration technique uses a very wide defect (shown on the right side of fig. 1) to simulate the erosion which would occur as representative of an unprotected material. Also, for an unprotected material, we know how much polymer should be eroded based on the atomic oxygen fluence and in-space erosion yield $\left(3.0 \times 10^{-24} \mathrm{~cm}^{3} /\right.$ atom for Kapton $\left.\mathrm{H}\right)$. That information then is used to determine the proportionally correct number of Monte Carlo atoms that should be brought into the model defect to produce an undercut cavity in which each cell is the length $(\mathrm{L}) \mathrm{cm}$ of an edge. Using this technique with the assumptions listed in table I, a Monte Carlo computational model undercut cavity was produced (see fig. 2) which represents the consequence of an atomic oxygen fluence of $7.15 \times 10^{21}$ atoms $/ \mathrm{cm}^{2}$ exposure on protected polyimide Kapton with a defect crack width of $4.16 \times 10^{-4} \mathrm{~cm}$. This undercut cavity and exposure is representative of samples returned from the Long Duration Exposure Facility. The thickness of the protective coating was assumed to be $2.45 \times 10^{-5} \mathrm{~cm}$.

To measure the ratio of in-space mass loss to ground laboratory mass loss, the number of oxygen atoms that reacted was measured using Monte Carlo calculations both as a function of energy and defect width. Based on information from these results, the ratio of in-space mass loss (at $4.5 \mathrm{eV}$ ) to ground laboratory mass loss (at arbitrary energies) was calculated as a function of ground laboratory facility directed beam energy.

\section{RESULTS AND DISCUSSION}

Based on the results of the Monte Carlo computational model the reaction parameters optimization is summarized in table $\mathrm{I}$. The probability of atomic oxygen reacting with a polymer upon the first impact is given in equation (2):

$$
\text { Probability of reaction upon initial impact }=k \exp -\left(E_{0} / E\right)
$$

where

$$
\begin{aligned}
& \mathrm{k}=0.1165 \\
& \mathrm{E}_{\mathrm{o}}=\text { activation energy }=0.26 \mathrm{eV} \\
& \mathrm{E}=\text { energy of atomic oxygen impact, } \mathrm{eV}
\end{aligned}
$$

The consequence of this dependence of probability of reaction on initial impact energy is that the number of Monte Carlo atoms required to be submitted at low energies is much greater than the number needed to produce the same amount of erosion as would occur with the same unprotected material at the high energies $(4.5 \mathrm{eV})$ of LEO as shown in figure 3.

The shape of the curve in figure 3 is obviously dependent on more than only the energy dependence of reaction probability because of the potential for scattered atom reactions. Thus, a variety of Monte Carlo computational model interaction parameters as shown in table I play a role in quantification of this calibration. 
Based on all the known interactions, it appears that directed beams at near room temperature thermal energies require absolute fluences two to three orders of magnitude higher than would be required if they were typical LEO $4.5 \mathrm{eV}$ energy.

Examination of the number of Monte Carlo computational model oxygen atoms that react as a function of energy for a constant effective fluence, results in a plot such as shown in figure 4 . As can be seen from figure 4, use of the calibration technique causes the number of reacted atoms to be approximately constant with energy for any specific width crack defect. This is in spite of the fact that the number of atoms required to be submitted at low energies is orders of magnitude higher than that at LEO energy. The slight variation in the number of reacted atoms with energy below $1 \mathrm{eV}$ is not understood at the present time. It may be due to the particular characteristics of interaction parameters at low energy where multiple collisions are encountered. Also shown in figure 4 for almost any size crack, the number of atoms reacted appears to be proportional with the crack width. This issue is more comprehensively explored in figure 5, which shows there is very little influence of protective coating defect width upon the fraction of Monte Carlo atoms that react. However, at low energy, orders of magnitude fewer atoms react than at orbital energies. The characteristic that narrow cracks behave similarly to wide cracks in terms of fraction of Monte Carlo atoms reacted, allows simplifying assumptions to be made concerning the projection of in-space mass loss based on ground laboratory mass loss data. It is not necessary to know the size distribution of protective coating defects because they behave similarly in terms of fraction of atoms reacted. Thus, the calibration from ground laboratory to in-space is dominated only by the energy dependence for the calibration of effective fluence which encompasses all the interaction parameters shown in table $I$.

Figure 6 is a plot of the in-space mass loss relative to ground laboratory mass loss as a function of energy for a fixed effective fluence of $7.15 \times 10^{21}$ atoms $/ \mathrm{cm}^{2}$, based on Monte Carlo modeling data using the calibration techniques previously described. This plot has a shape similar to that in figure 4 . A specific width of $4.16 \times 10^{-4} \mathrm{~cm}$ width defect in the protective coating was used for this simulation based on the results in figure 5 , however, any width defect could have been used. The reference energy for the in-space mass loss was $4.5 \mathrm{eV}$. The term "mass loss" is used as opposed to "atoms reacted" because mass or volume is considered a matter of prime concern and mass loss or volume loss is proportional to the number of oxygen atoms reacted.

As can be seen from figure 6, the Monte Carlo model predicted ratio of in-space mass loss to ground laboratory mass loss has only small variations from unity if the Kapton $\mathrm{H}$ effective fluence calibrations for operating directed beam ground laboratory facilities are used. The predicted in-space mass loss is, at most, 10 percent higher and not more than 30 percent lower than ground laboratory mass loss test results would indicate. For highly elliptical LEO missions, energies above $4.5 \mathrm{eV}$ would exist which is the purpose of gathering predictions up to $8 \mathrm{eV}$ as shown in figure 6. It is expected that the shape of this in-space to ground laboratory mass loss curve shown in figure 6 would be sensitive to material differences whose atomic oxygen interaction characteristics differ from those listed in table I. However, atomic oxygen interactions with Kapton $\mathrm{H}$ have been found to be quite similar to many other hydrocarbon materials. Thus, one would be inclined to believe that using effective fluence measurements with whatever specific material is in question, coupled with in-space data from that material, would allow reasonable confidence that the in-space mass loss would quite closely replicate the results of directed beam ground laboratory testing to the same effective fluence.

\section{SUMMARY}

A Monte Carlo computational model was used to explore the energy dependence of atomic oxygen reacting with polyimide Kapton $\mathrm{H}$ at sites of crack defects in protective coatings. The model was used in a calibrated form based on effective atomic oxygen fluence. Using Monte Carlo model atomic oxygen interaction parameters based on replication of undercut cavities experimentally measured from the Long Duration Exposure Facility, atomic oxygen reaction as a function of energy and crack width was explored. Because the fraction of reacted oxygen atoms relative to submitted atoms is essentially constant with defect width and only dependent upon impact atom energy, the ratio of in-space mass loss to ground laboratory mass loss as a function of energy for any size distribution of protective coating defects on polyimide Kapton $\mathrm{H}$ can be calculated. Results indicate that in-space mass loss should be at most 10 percent higher and not less than 30 percent lower than directed beam ground laboratory testing would predict, based on tests conducted to the same effective fluence as in space. 


\section{REFERENCES}

1. Leger, L.J., "Oxygen Atom Reaction of Shuttle Materials at Orbital Altitudes," NASA TM-8246, 1982.

2. Banks, B.A., Mirtich, Mirtich, M.J., Rutledge, S.K., and Swec, D.M., "Sputtered Coatings for Protection of Spacecraft Polymers," NASA TM-83706, 1984.

3. Coulter, D. R., Liang, R.H., Chung, S.Y., Smith, K.O., and Gupta, A., "O-atom Degradation Mechanisms of Materials," Proceedings of the NASA Workshop on Atomic Oxygen Effects, David E. Brinza, editor, JPL Publication 87-14, Nov. 1986.

4. Silverman, E., "Space Environmental Effects on Spacecraft: LEO Materials Selection Guide," NASA CR-4661, Aug. 1995.

5. Banks, B.A., "The Use of Fluoropolymers in Space Applications," Modern Fluoropolymers, edited by John Schiers, John Wiley \& Sons, 1997.

6. Connell, J.W., Hergenrother, P.M., and Smith, J.G., "Oxygen Plasma-Resistant Phenylphosphene Oxidecontaining Polyimides," Polymer, vol. 36, 1995, p. 5.

7. Rutledge, S.K., and Mihelcic, J.M., "The Effect of Atomic Oxygen on Altered and Coated Kapton Surfaces for Spacecraft Applications in Low Earth Orbit," Proceedings of the Materials Degradation in Low Earth Orbit symposium at the 119th annual meeting of the TMS in Anaheim, CA, Feb. 17-22, 1990, (V. Srinivasan and B. Banks, eds.)

8. Rutledge, S.K., and Olle, R.M., "Space Station Freedom Solar Array Blanket Coverlay Atomic Oxygen Durability," Proceedings of the 38th Int'l. SAMPE Symposium, May 10-13, 1993, pp. 679-693.

9. Jaworske, D.A., de Groh, K.K., Podijil, G.M., McCollum, T.A., and Anzic, J., "Leveling Coatings for Reducing Atomic Oxygen Defect Density in Graphite Fiber Epoxy Composites," J. of IES, May/June 1994, pp. 26-31.

10. Forkapa, M.J., Stidham, C.R., Banks, B.A., Rutledge, S.K., Ma, D.H., and Sechkar, E.A., "Atomic Oxygen Durability Testing of International Space Station Solar Array Validation Coupon," NASA TM-107212, presented at the 3rd International Space Conference on Protection of Materials and Structures from the LEO Space Environment," University of Toronto, Toronto, Canada, April 25-26, 1996.

11. Rutledge, S.K., and Milhelcic, J.A., "Undercutting of Defects in Thin Film Protective Coatings on Polymer Surfaces Exposed to Atomic Oxygen," NASA TM-101986, 1989.

12. Banks, B.A., Rutledge, S.K., de Groh, K.K., Auer, B.M., Hill, C.M., "Atomic Oxygen Protective Coatings," Proceedings of the NATO Advanced Study Institute Conference, Pitlochry, Scotland, July 7-19, 1991.

13. Banks, B.A., Rutledge, S.K., de Groh, K.K., Auer, B.M., Mirtich, M.J., Gebauer, L., Hill, C.M., Lebed, R.F., "LDEF Spacecraft, Ground Laboratory and Computational Modeling Implications on Space Station Freedom Solar Array Materials and Surfaces Durability," Proceedings of the IEEE Photovoltaic Specialists Conference, Las Vegas, Nevada, October 7-11, 1991.

14. de Groh, K.K., and Banks, B.A., "Atomic Oxygen Undercutting of Long Duration Exposure Facility Aluminized Kapton Multi-Layer Insulation," J. Spacecraft \& Rockets, 31:45, pp. 656-664, Aug. 1994.

15. Banks, B.A., Rutledge, S.K., de Groh, K.K., Stidham, C.R., Gebauer, L., and LaMoureaux, C., "Atomic Oxygen Durability Evaluation of Protected Polymers using Thermal Energy Plasma Systems," NASA TM-106855, Proceedings of the International Conference on Plasma Synthesis and Processing of Materials, sponsored by the Metallurgical Society, Denver, Co., Feb. 21-25, 1993.

16. Banks, B.A., Stueber, T.J., "Monte Carlo Computational Techniques for Prediction of Atomic Oxygen Erosion of Materials," Proceedings of the NATO Advanced Research Workshop on Computer Modeling of Electronic and Atomic Processes in Solids (Space and Electronic Materials), Wroclaw, Poland, May 1996.

17. Banks, B.A., de Groh, K.K., Rutledge, S.K., DiFillipo, F.J., "Prediction of In-Space Durability of Protected Polymers Based on Ground Laboratory Thermal Energy Atomic Oxygen," NASA TM-107209, Proceedings of the 3rd International Space Conference on Protection of Materials and Structures from the LEO Space Environment," University of Toronto, Toronto, Canada, April 25-26, 1996.

18. Banks, B.A., Stueber, T.J., Snyder, S.A., Rutledge, S.K., and Norris, M.J., "Atomic Oxygen Erosion Phenomena," presented at the American Institute of Aeronautics and Astronautics, Space Programs Conference, Huntsville, AL, Sept. 23-25, 1997. 
TABLE I-COMPUTATIONAL MODEL PARAMETERS AND REFERENCE VALUES FOR LEO ATOMIC OXYGEN INTERACTION WITH KAPTON.

\begin{tabular}{|l|l|}
\hline Initial impact reaction probability & 0.11 \\
\hline Activation energy $\mathrm{E}_{0}, \mathrm{eV}$, for energy dependent reaction probability form & 0.26 \\
\hline $\begin{array}{l}\text { Atomic oxygen reaction probability angle of impact dependence exponent, } \mathrm{n}, \text { in } \cos ^{\mathrm{n}} \boldsymbol{\theta} \text { angular } \\
\text { dependence of atomic oxygen erosion yield where } \theta \text { is the angle between the arrival direction and } \\
\text { the local surface normal }\end{array}$ & 0.5 \\
\hline Probability of atomic oxygen recombination upon impact with protective coating & 0.13 \\
\hline Probability of atomic oxygen recombination with polymer & 0.24 \\
\hline Fractional energy loss upon impact with polymer & 0.28 \\
\hline $\begin{array}{l}\text { Degree of specularity as opposed to diffuse scattering of atomic oxygen upon non-reactive impact } \\
\text { with protective coating where } 1=\text { fully specular scattering and } 0=\text { fully diffuse scattering }\end{array}$ & 0.4 \\
\hline $\begin{array}{l}\text { Degree of specularity as opposed to diffuse scattering of atomic oxygen upon non-reactive impact } \\
\text { with polymer where } 1=\text { fully specular scattering and } 0=\text { fully diffuse scattering }\end{array}$ & 0.035 \\
\hline Temperature for thermally accommodated atom $(\mathrm{K})$ & 300 \\
\hline Bounce limit & 25 \\
\hline $\begin{array}{l}\text { Thermally accommodated energy/actual atom energy for atoms to be assumed thermally } \\
\text { accommodated }\end{array}$ & 0.9 \\
\hline Atomic oxygen average arrival direction with respect to initial surface normal, degrees & 0 \\
\hline Initial oxygen atomic energy, eV & 4.5 \\
\hline Thermosphere temperature for atomic oxygen, ${ }^{\circ} \mathrm{K}$ & 1000 \\
\hline $\begin{array}{l}\text { Atomic oxygen arrival plane relative to Earth for a Maxwell-Boltzmann atomic oxygen temperature } \\
\text { distribution and an orbital inclination of } 28.5^{\circ} \text {. }\end{array}$ & Horizontal \\
\hline
\end{tabular}

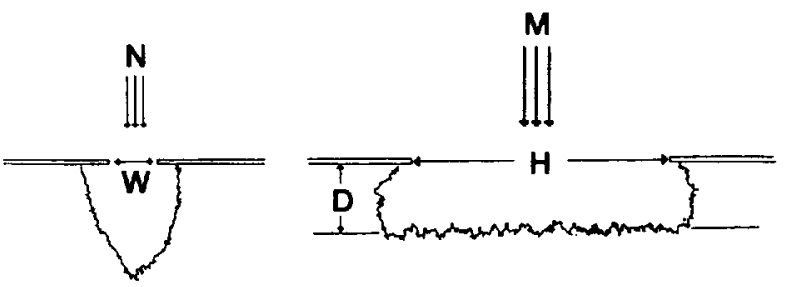

Figure 1.-Monte Carlo computational model calibration.

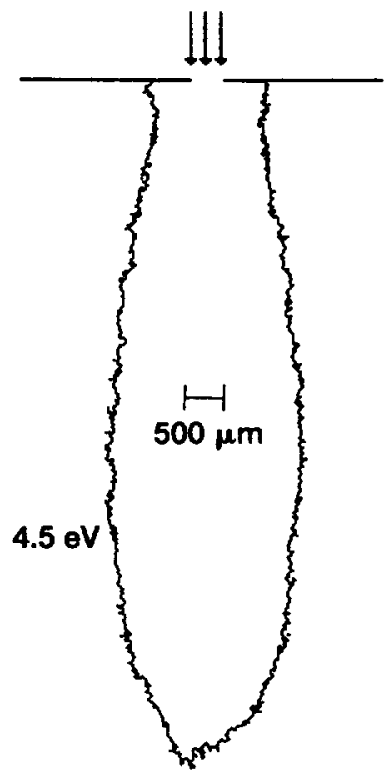

Figure 2.-Monte Carlo computational model predicted undercut cavity for a fluence of $7.15 \times 10^{21}$ atoms $/ \mathrm{cm}^{2}$ exposure at a defect width of $4.6 \times 10^{-4}$ $\mathrm{cm}$ in a protective coating on Kapton $\mathrm{H}$ polyimide. 


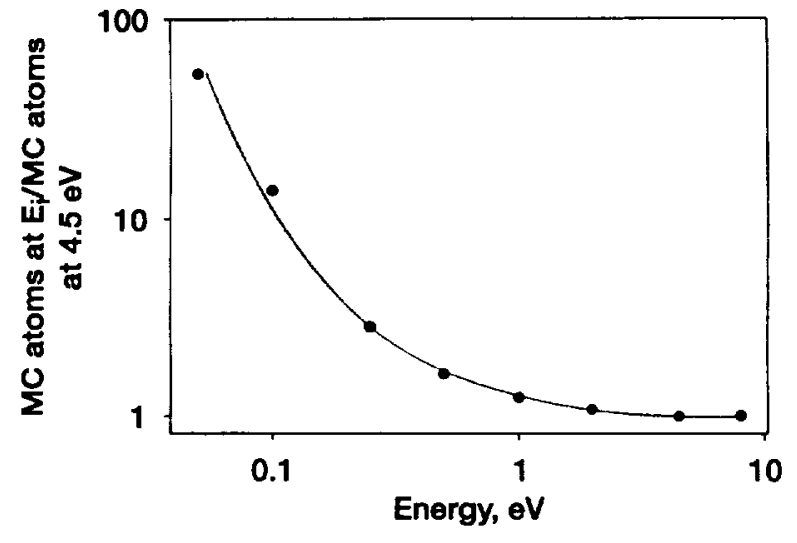

Figure 3.-Ratio number of Monte Carlo (MC) computational model atoms required at energy $E_{i}$ to those at $4.5 \mathrm{eV}$ to cause the same amount of erosion for unprotected Kapton $\mathrm{H}$.

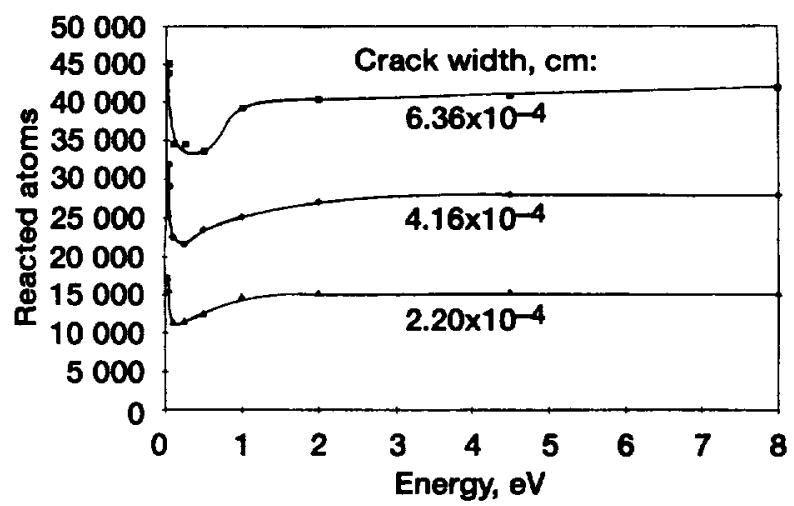

Figure 4.-Number of Monte Carlo (MC) computational model atoms that react as a function of energy for 3 widths of defects in the protective coating and a constant effective fluence of $7.15 \times 10^{21}$ atoms $/ \mathrm{cm}^{2}$.

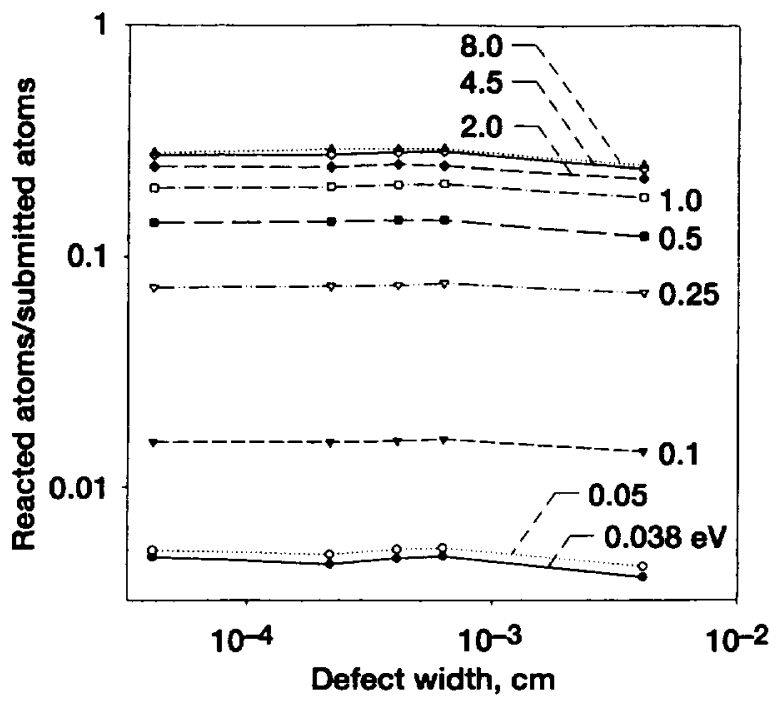

Figure 5.-Ratio of reacted Monte Carlo atoms to submitted Monte Carlo atoms as a function of protective coating crack width for various energy for a constant effective fluence of $7.15 \times 10^{21}$ atoms $/ \mathrm{cm}^{2}$.

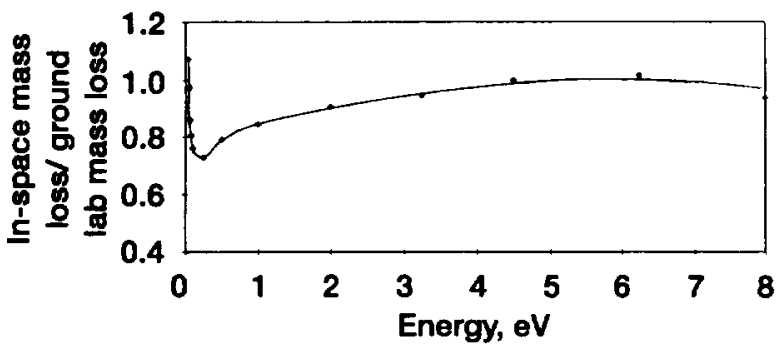

Figure 6.-In-space mass loss relative to ground laboratory mass loss as a function of energy for a fixed effective fluence of $7.15 \times 10^{21}$ atoms $/ \mathrm{cm}^{2}$, based on Monte Carlo modeling data using the calibration techniques. 


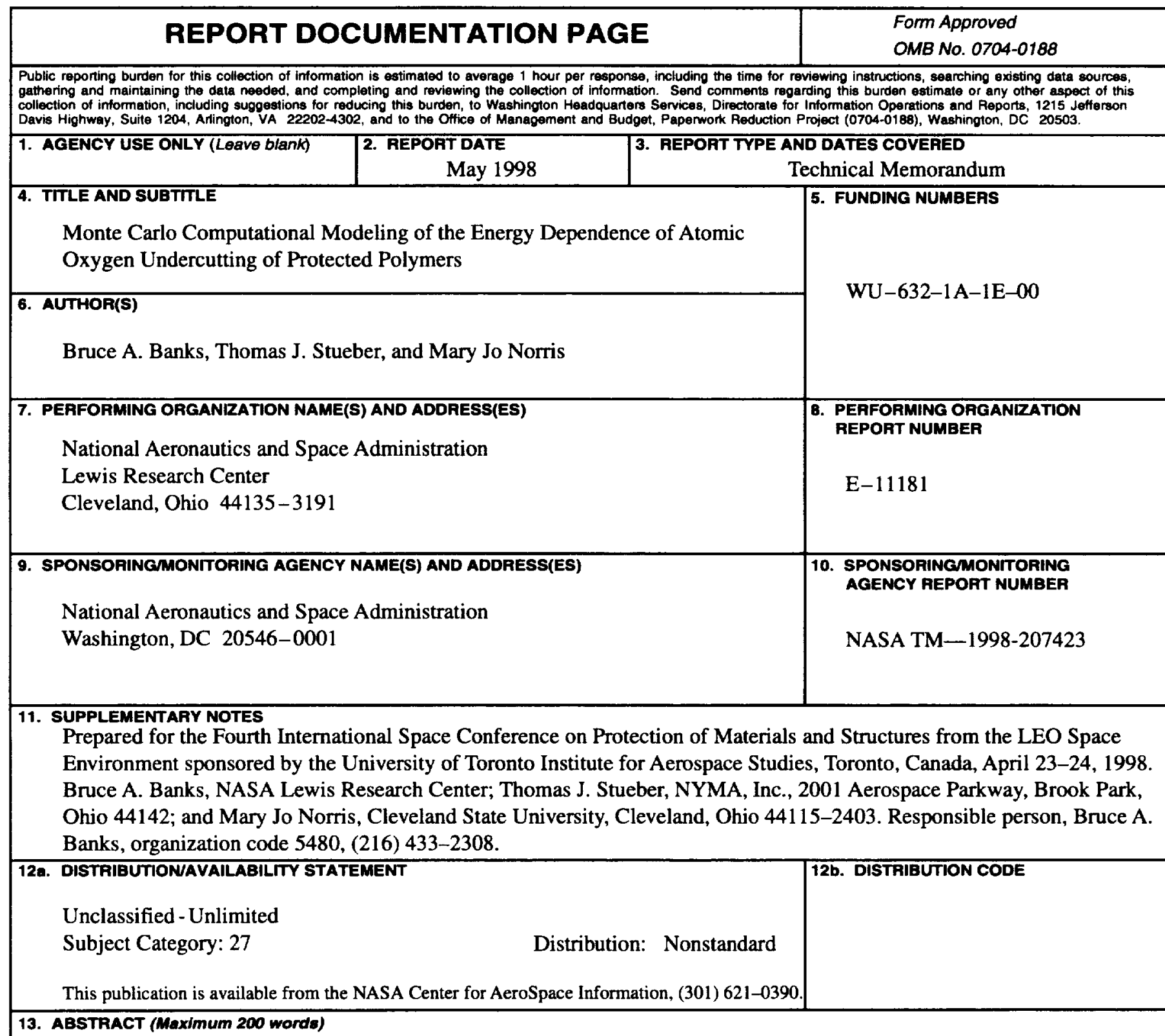

A Monte Carlo computational model has been developed which simulates atomic oxygen attack of protected polymers at defect sites in the protective coatings. The parameters defining how atomic oxygen interacts with polymers and protective coatings as well as the scattering processes which occur have been optimized to replicate experimental results observed from protected polyimide Kapton on the Long Duration Exposure Facility (LDEF) mission. Computational prediction of atomic oxygen undercutting at defect sites in protective coatings for various arrival energies was investigated. The atomic oxygen undercutting energy dependence predictions enable one to predict mass loss that would occur in low Earth orbit, based on lower energy ground laboratory atomic oxygen beam systems. Results of computational model prediction of undercut cavity size as a function of energy and defect size will be presented to provide insight into expected in-space mass loss of protected polymers with protective coating defects based on lower energy ground laboratory testing.

\begin{tabular}{|c|c|c|c|}
\hline \multicolumn{3}{|l|}{ 14. SUBJECT TERMS } & 15. NUMBER OF PAGES \\
\hline \multicolumn{3}{|c|}{ Monte Carlo computational modeling; Atomic oxygen; Polyimide Kapton } & \begin{tabular}{|r}
13 \\
16. PRICE CODE
\end{tabular} \\
\hline & & & $\mathrm{A} 03$ \\
\hline $\begin{array}{l}\text { 17. SECURITY CLASSIFICATION } \\
\text { OF REPORT }\end{array}$ & $\begin{array}{l}\text { 18. SECURTYY CLASSIFICATION } \\
\text { OF THIS PAGE }\end{array}$ & $\begin{array}{l}\text { 19. SECURTYY CLASSIFICATION } \\
\text { OF ABSTRACT }\end{array}$ & 20. LIMTTATION OF ABSTRACT \\
\hline Unclassified & Unclassified & Unclassified & \\
\hline
\end{tabular}

